\title{
Localization of the CyanoP binding site on photosystem II by surface plasmon resonance spectroscopy
}

\section{Kai U. Cormann, Maik Bartsch, Matthias Rögner and Marc M. Nowaczyk*}

Plant Biochemistry, Ruhr University Bochum, Bochum, Germany

\section{Edited by:}

Richard Sayre, New Mexico

Consortium and Los Alamos National

Labs, USA

\section{Reviewed by:}

Jin Chen, Michigan State University, USA

Xia Wu, University of Washington, USA

\section{*Correspondence:}

Marc M. Nowaczyk, Plant Biochemistry, Ruhr University Bochum, Universitätsstraße 150, 44801 Bochum, Germany e-mail:marc.m.nowaczyk@rub.de
Photosystem II (PSII), a large multi subunit membrane protein complex localized in the thylakoid membrane of cyanobacteria and chloroplasts, is the only known enzyme that catalyzes the light-driven oxidation of water. In addition to the membrane intrinsic part of PSII, efficient oxygen evolution requires soluble protein subunits at its luminal interface. In contrast to the detailed crystal structure of the active cyanobacterial complex the characterization of intermediate PSII species related to its assembly and repair is hampered by their instability or low abundance. As most structural variations of the corresponding PSII species are based on a different set of protein factors bound to the luminal interface of the complex we developed a system for interaction analysis between PSII and its soluble interaction partners based on surface plasmon resonance (SPR) spectroscopy. The assay was validated by the correct localization of the extrinsic PSII proteins PsbO, PsbV, and PsbU on the luminal PSIl surface and used to determine the unknown binding position of CyanoP, the cyanobacterial homolog of higher plant PsbP. The CyanoP binding site was clearly localized in the center of PSII at a position, which is occupied by the PsbO subunit in mature PSII complexes. Consistently, we demonstrate selective binding of CyanoP to an inactive PSII assembly intermediate that lacks the extrinsic subunits PsbO, PsbV, and PsbU. These findings suggest, that CyanoP functions in the dynamic lifecycle of PSII, possibly in the association of CP47 and CP43 or in photoactivation of the oxygen-evolving complex.

Keywords: photosynthesis, photosystem II, CyanoP, PsbP, surface plasmon resonance (SPR), assembly factor, photoactivation

\section{INTRODUCTION}

Photosystem II (PSII) catalyzes one of nature's key reactions: the light-driven oxidation of water. Remarkable advances in X-ray crystallography of cyanobacterial PSII improved the structural models of this multisubunit pigment-protein complex to a resolution of $1.9 \AA$ (Umena et al., 2011). In total 19 protein subunits and multiple cofactors like chlorophylls, carotenoids, lipids, metal ions, and the oxygen-evolving complex (OEC), the catalytic center of the water-splitting reaction, have been elucidated in this recent model. Out of the 19 subunits, 16 proteins are embedded in the thylakoid membrane, whereas the three extrinsic proteins PsbO, PsbV, and PsbU are solvent accessible, protruding out into the thylakoid lumen. Notably, the crystal structure depicts only one out of various PSII complexes. Those which represent assembly and repair intermediates of the PSII lifecycle (Komenda et al., 2012), are known to contain a makeup of extrinsic factors different from that of the fully assembled complex (Nowaczyk et al., 2006; Rengstl et al., 2011). For instance, Psb27, a lipoprotein associated with CP43 (Liu et al., 2011), is only transiently bound to the PSII complex; it appears to have a role in the assembly and repair process of PSII after photodamage (Nowaczyk et al., 2006; Grasse et al., 2011). Another example includes CtpA, a sequence specific protease, which cleaves a C-terminal extension from the D1-precursor subunit in the early phase of PSII biogenesis (Anbudurai et al., 1994), a prerequisite for the assembly of the OEC.
In addition, the composition of the extrinsic subunits has changed over the course of evolution of photosynthetic organisms (Nagarajan and Burnap, 2012). In contrast to PsbO, which is common for all photoautotrophs, the presence of PsbV and PsbU is restricted to cyanobacteria (Shen et al., 1992), red algae (Enami et al., 1995), and diatoms (Nagao et al., 2010b), while PSII of green algae (Suzuki et al., 2003) and higher plants (Murata et al., 1984) contains stoichiometric amounts of PsbP and PsbQ. Interestingly, cyanobacterial homologs of these subunits (CyanoP and CyanoQ, respectively) have been found in PSII preparations from Synechocystis sp. PCC 6803 (Thornton et al., 2004), and CyanoQ was shown to be specific for highly active PSII complexes (Roose et al., 2007). However, both proteins are missing in PSII purified from Thermosynechococcus vulcanus, which has been crystallized (Umena et al., 2011).

The impact of these various compositions on the PSII structure is difficult to elucidate due to their low abundance, transient nature or instability. In particular, the structural characterization of CyanoP PSII complexes is hampered by their low abundance in cyanobacterial PSII preparations (Thornton et al., 2004; Ishikawa et al., 2005). This may be due to loss during purification (Ishikawa etal., 2005) or the small amount of CyanoP containing PSII species (Thornton et al., 2004). In contrast to the well characterized function of higher plant PsbP in maintaining PSII activity (Ifuku et al., 2005; Yi et al., 2007; Ido et al., 2012) and regulating 
the binding of PsbQ (Kakiuchi et al., 2012), the physiological role of CyanoP is still unknown. This is surprising as CyanoP represents the phylogenetic origin of the whole PsbP superfamily (Sato, 2010) and the structures of CyanoP (Michoux et al., 2010; Jackson et al., 2012) and PsbP (Ifuku et al., 2004) share a high degree of similarity. Here we introduce an in vitro assay based on surface plasmon resonance (SPR) spectroscopy for the localization of transiently bound proteins on large (membrane) protein complexes. While PsbP is a structural component of the active PSII complex in green algae and plants, our results indicate a role of CyanoP in the dynamic PSII lifecycle, presumably in the association of CP47 and CP43 or in photoactivation of the OEC.

\section{MATERIALS AND METHODS POLYMERASE CHAIN REACTION (PCR) AND MOLECULAR CLONING}

Gene sequences from Thermosynechococcus elongatus coding for CyanoP, CyanoQ, PsbO, PsbU, PsbV, and Psb27 without signal peptide and the luminal domains of precursor D1 (pD1), mature D1 (mD1), D1 a-loop (D1a), D1 peptide (D1pep), D2, CP43, CP47, and PsbE were amplified by PCR using oligonucleotides summarized in Table S1. Details of the cloning procedure for the generation of expression templates for the immunity protein 7 (Im7) fusion proteins, CyanoP, PsbO, and PsbV are given in the supplementary material.

\section{CELL-FREE PROTEIN EXPRESSION}

Cell-free expression of the Im7 tagged luminal domains was done according to the manufacturer's instructions using the RTS100system (5Prime). Briefly, 500 ng of expression plasmid were added to the reaction mixture. The expression reaction was performed by incubation for $6 \mathrm{~h}$ at $30^{\circ} \mathrm{C}$ under slight shaking $(100 \mathrm{rpm})$. Aliquots were stored at $-80^{\circ} \mathrm{C}$.

\section{HETEROLOGOUS PROTEIN OVEREXPRESSION AND PURIFICATION}

Heterologous overexpression of CyanoP, PsbO, and Im7 fusion proteins of PsbO, PsbU, Psb27, CyanoP, and CyanoQ was performed with Escherichia coli Overexpress C43 cells (Lucigen) and the corresponding expression vector derived from pIVEX2.4d (5Prime). A more detailed description of the expression and purification procedure is given in the supplementary material. Expression of PsbV was based on a previously published protocol (Andrews et al., 2005). Details on the purification of the streptagged protein are given in the supplementary material. Expression and purification of DNase E7 was carried out according to Hosse et al. (2009).

\section{MASS SPECTROMETRY}

Sample preparation and mass spectrometric analysis for identification of the Im7 fusion proteins was done according to Nowaczyk et al. (2011). An E. coli K12 protein database supplemented with the sequences for the $\operatorname{Im} 7$ fusion proteins or a T. elongatus protein database was used in this approach.

\section{SPR EXPERIMENTS}

All SPR measurements were performed with a Biacore3000 instrument using CM5 sensor chips (both GE Healthcare). Preparation of DNase E7 coated surfaces was done according to Hosse etal. (2009). Additionally, the surface was conditioned with two consecutive $1 \mathrm{~min}$ injections of Gentle Elution Buffer (Thermo Scientific) at a flow rate of $60 \mu \mathrm{l} / \mathrm{min}$. Details of the on-chip purification of $\operatorname{Im} 7$ fusion proteins and the SPR interaction analysis are given in the supplementary material.

\section{PREPARATION OF PSII AND RECONSTITUTION EXPERIMENTS}

Preparation of PSII complexes from T. elongatus (wildtype) was done according to Kuhl et al. (2000), Nowaczyk et al. (2006). For reconstitution of PSII with recombinant CyanoP inactive monomeric PSII and highly active dimeric PSII (Nowaczyk et al., 2006; both at $0.1 \mathrm{mg} / \mathrm{ml}$ chlorophyll) were incubated with a twofold molar excess of CyanoP $\left(15 \mathrm{~min} ; 4^{\circ} \mathrm{C}\right)$ in buffer A $\left(20 \mathrm{mM}\right.$ MES, $10 \mathrm{mM} \mathrm{MgCl}_{2}, 10 \mathrm{mM} \mathrm{CaCl}_{2}, 0.03 \%$ $(\mathrm{w} / \mathrm{v})$ n-Dodecyl $\beta$-D-maltoside, $\mathrm{pH}$ 6.5). Unbound CyanoP was removed by three washing steps with $250 \mu \mathrm{l}$ buffer $\mathrm{A}$ using centrifugal filter devices (Microcon YM100, Millipore; $6.000 \mathrm{~g}, 10 \mathrm{~min}, 4^{\circ} \mathrm{C}$ ). Reconstitution experiments were performed as two independent biological replicates using different PSII preparations.

\section{RESULTS}

\section{THE LUMINAL INTERFACE OF PSII CAN BE MIMICKED BY RECOMBINANT PROTEIN DOMAINS}

In the thylakoid lumen, most of the binding interface for the extrinsic subunits of PSII and the assembly factors is provided by the solvent exposed e-loops of CP43 and CP47, by the D1 a-loop, as well as by the C-termini of D1, D2, and PsbE (Figure 1A; Table 1). Hence, we decided to express these domains from T. elongatus (Figure 1B) as recombinant proteins and immobilize them on an SPR sensor surface to determine their affinity for their putative interaction partners. This approach is based on a previously reported capture system (Hosse et al., 2009) utilizing the tight interaction between the nuclease domain of colicinE7 (DNase E7) and its inhibitor, Im7. The latter was fused via a hydrophilic and flexible linker sequence (GGSG) to the N-terminus of the CP43/CP47 e-loops, D1a and the C-terminal domains of $\mathrm{pD} 1$, $\mathrm{mD} 1, \mathrm{D} 1 \mathrm{pep}, \mathrm{D} 2$, and PsbE (Figure 2A) allowing stable and selective immobilization of the fusion construct on DNase E7 coated surfaces (Hosse et al., 2009). Moreover, the structure of DNase E7 in complex with Im7 (Ko et al., 1999) suggests that the immobilized PSII domain is completely accessible for soluble proteins (Figure 2A). To ensure that the domains adopt a conformation which allows specific binding of their interaction partners, an extrinsic PSII subunit with known binding position (e.g., PsbV, PsbO) can be used as positive or negative control. This subunit is first injected over a reference surface coated with DNase E7 in complex with $\operatorname{Im} 7$ in order to check for unspecific binding to the sensor surface (Figures 2B,C). Subsequently, the same sample is exposed to an analogous surface with either an interacting (positive control) or non-interacting (negative control) Im7-PSII fusion protein. As shown in the following paragraphs this carefully referenced setup enables the precise detection of specific protein-protein interactions even if the corresponding binding affinities are in the intermediate micromolar range. 
A

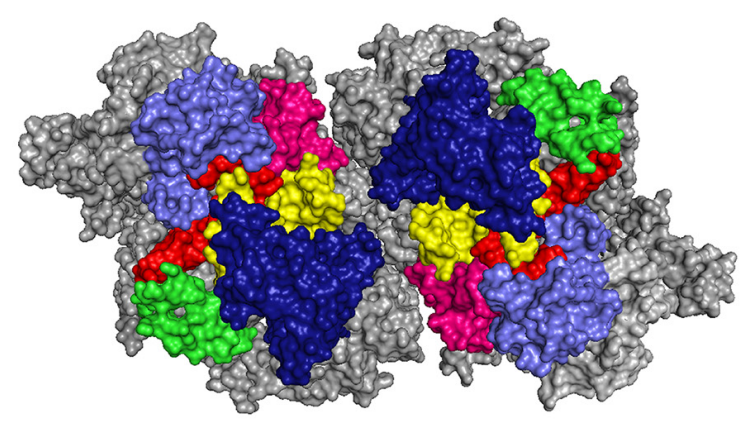

B

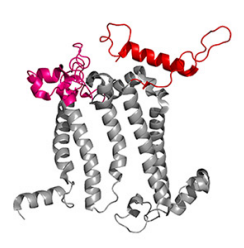

D1

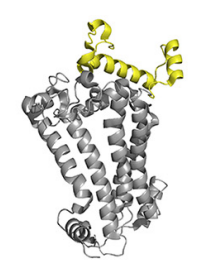

D2

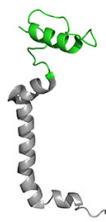

PsbE

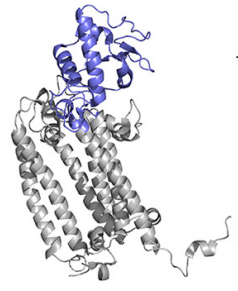

CP43

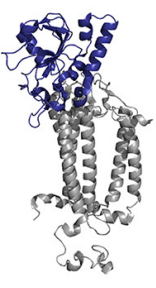

CP47
FIGURE 1 |The luminal interaction interface of PSII. (A) Top view of the luminal side of dimeric PSII without extrinsic subunits (Umena etal., 2011). The e-loops of CP43 (light blue), CP47 (dark blue), the a-loop of D1 (pink) and the C-terminal domains of D1 (red), D2 (yellow), and PsbE (green) represent the major interaction sites for soluble subunits. (B) Cartoon showing the individual PSII subunits of which the colored domains can be expressed as recombinant proteins to be used for biomolecular interaction analysis with extrinsic PSII subunits or assembly factors.
Table 1 | Nomenclature of different D1 domains.

\begin{tabular}{lll}
\hline Abbreviation & Domain & Residues (PsbA1) \\
\hline mD1 & $\begin{array}{l}\text { C-terminal D1 domain in } \\
\text { its processed form }\end{array}$ & N295 - A344 \\
& $\begin{array}{l}\text { C-terminal D1 domain } \\
\text { with precursor peptide }\end{array}$ & N295 - G360 \\
& D1 a-loop & A54 - O113 \\
D1a & C-terminal peptide of & H332-A344 \\
D1pep & processed D1 & \\
& & \\
\hline
\end{tabular}

\section{Im7-FUSION PROTEINS OF THE LUMINAL PSII DOMAINS CAN BE PURIFIED ON THE SPR SENSOR SURFACE}

The luminal PSII domains fused to $\operatorname{Im} 7$ were expressed in a cellfree system (RTS100, 5Prime) in order to avoid degradation of the truncated constructs, which was confirmed by SDS-PAGE and MS analysis (Figures S1 and S2). As cell-free expression yielded only a low amount of recombinant PSII domains, a sensor surface with immobilized DNase E7 (approximately 3500 response units, RU) was used as affinity matrix for the Im7-tagged constructs. The remarkable affinity and specificity of the Im7-E7 system enables on-chip purification and immobilization in one single step (Figure 2B) as confirmed by a control implemented in every experiment: the fact that an injection of a reaction mixture without template DNA over the reference surface did not affect the baseline level excludes unspecific binding to the sensor surface. In contrast, injection of a sample expressing the Im7-CP43 fusion protein increased the baseline on the active surface of the second flow cell by 510 RU. As the composition of both cell-free expression mixtures is identical - besides the presence of Im7-CP43 this indicates a close to $100 \%$ purity of the Im7-tagged protein on the active surface. Also, in order to ensure maximal comparability between reference and active cell, both surfaces were saturated with purified Im7. Surface preparations with the other Im7-PSII fusion proteins showed identical purity and varied only in the amount of captured protein on the active surface due to differences in expression efficiency and molecular mass of the fused domains.

\section{RECOMBINANT PSII DOMAINS ARE SUITABLE FOR IDENTIFICATION OF THE MAJOR BINDING INTERFACES OF EXTRINSIC PSII SUBUNITS}

The feasibility of our SPR based approach for mapping of protein binding sites on large (membrane) protein complexes was verified by analysis of the well-known binding position of PsbV and PsbO at the luminal surface of PSII. The PSII crystal structure (Umena etal., 2011) shows a major contact of PsbV with the CP43 e-loop and minor interactions with the C-terminus of D1 and the PsbU subunit. Indeed, a concentration dependent and reproducible binding to immobilized Im7-CP43 at PsbV concentrations $\geq 1 \mu \mathrm{M}$ was observed (Figure 3). A plot of the steady state responses against the corresponding PsbV concentrations was fitted accurately to a one site binding isotherm yielding an affinity constant of $26 \pm 2 \mu \mathrm{M}$. Although the reference surface can be used as intrinsic negative control for unspecific binding, the binding of PsbV to the e-loop of CP47, D1a and D2 was used as an additional control, yielding no binding except for a weak signal at $50 \mu \mathrm{M}$ PsbV. In contrast to CP43, binding of PsbV to $\mathrm{mD} 1$ is similar to the unspecific interactions with the e-loop of CP47 and D1a, suggesting a weak contribution of D1 to the association of PsbV. The binding responses of PsbU, the third subunit with contact interfaces to PsbV (Umena et al., 2011), can be fitted to a one site binding model, but the calculated dissociation constant of $146 \mu \mathrm{M}$ is $>$ twofold higher than the highest concentration used and thus represents a rough estimate of the exact value $\left(K_{\mathrm{D}} \approx 150 \mu \mathrm{M}\right)$. This intermediate affinity is in agreement with data showing an enhancement of PsbU-binding to PSII by PsbV, albeit PsbO is additionally required for a complete association 
A

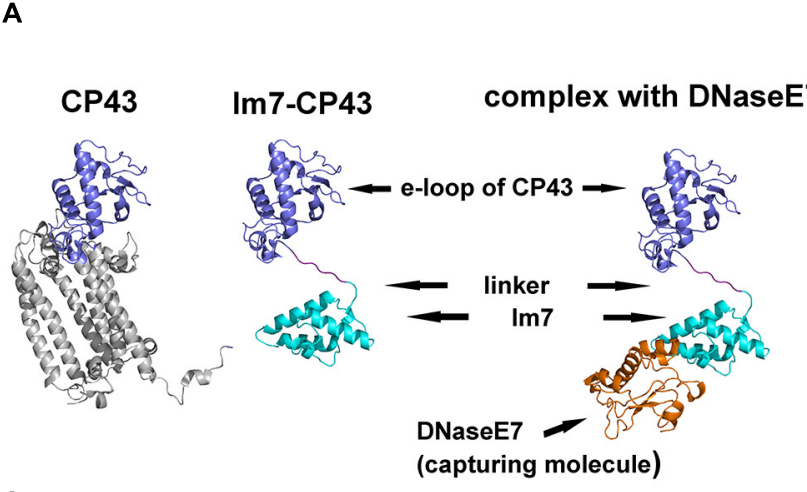

C
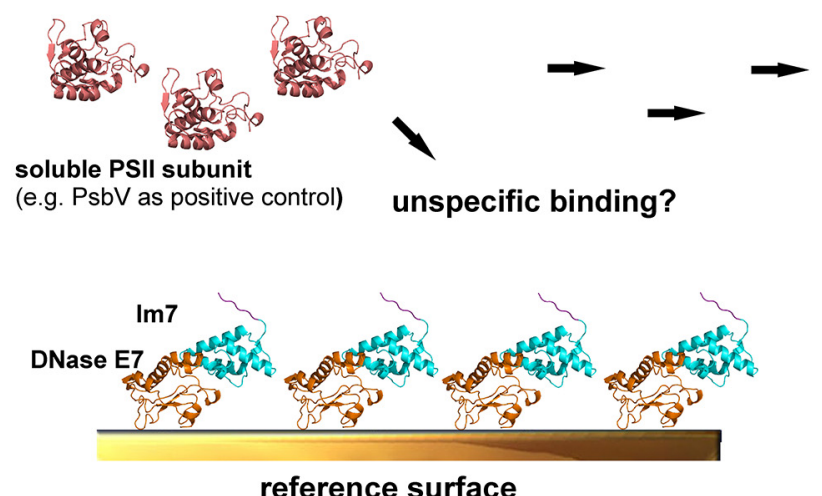

FIGURE 2 | Experimental approach for mapping the binding sites of extrinsic subunits and assembly factors of PSII. The structural models are based on the crystal structures of PSII (Umena etal., 2011; pdb code: 3ARC) and the complex between DNase E7 and Im7 (Ko etal., 1999; pdb code: 7CEI). (A) Structural models for CP43, the e-loop of CP43 tagged with Im7 and the Im7-CP43 fusion protein bound to DNase E7. The Im7-tag (cyan) and DNase E7 (orange) are located in a position occupied by the transmembrane helices of native CP43 (gray), and thus neither the tag nor DNase E7 interfere with the binding of soluble interaction partners to the e-loop (light blue). (B) Preparation of active (red) and reference surface (blue) for surface plasmon resonance (SPR) interaction analysis. Unspecific binding of the reaction mixture to the surface is checked by injecting a 1000 -fold dilution over the reference cell, upon which the signal returned to the baseline level. This indicates the absence of unspecific binding. In contrast, injection of a reaction mixture (identical dilution) expressing Im7-CP43 results in stable

\section{B}
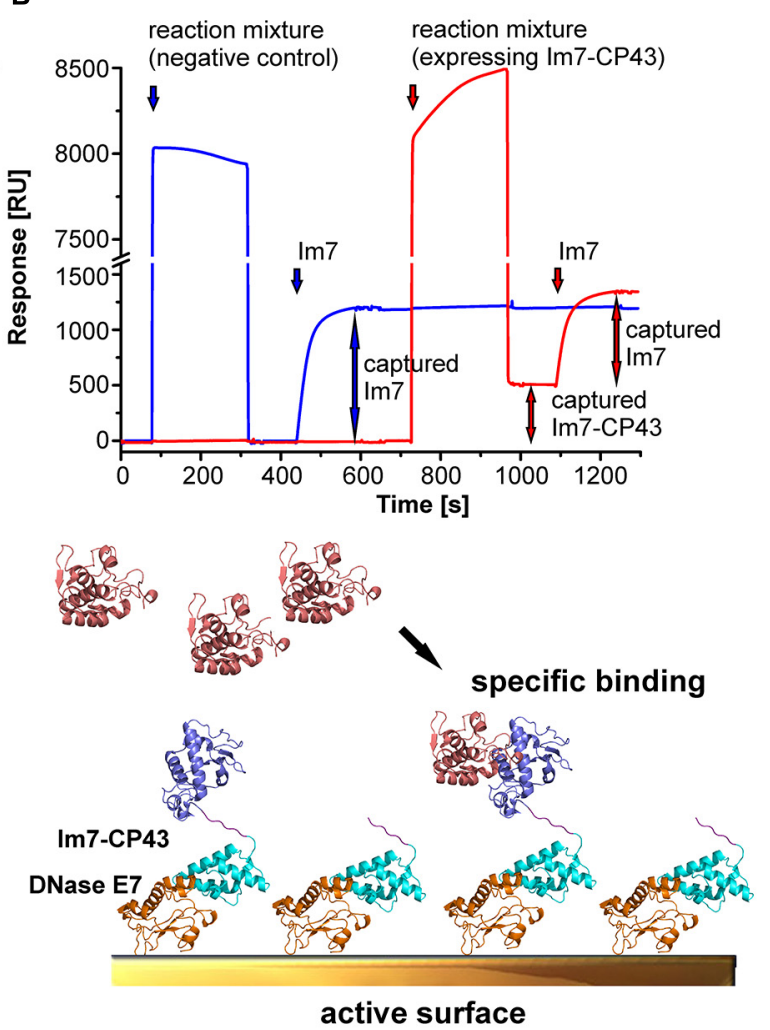

immobilization of $510 \mathrm{RU}$ of fusion protein. Accordingly, the purity of the immobilized PSII domain on the SPR surface is close to $100 \%$. Finally, both surfaces were saturated with purified $\operatorname{Im} 7(200 \mathrm{nM})$ to achieve maximal comparability between reference and active surface; division of the $y$-axis (high bulk signal caused by the high ionic strength of the immobilization buffer) should be considered. (C) Schematic structures of the surfaces prepared in (B), which were used as positive control for SPR interaction analysis between Im7-CP43 [colors according to (B)] and PsbV (salmon). DNase E7 is covalently bound to the sensor surface, allowing stable immobilization of $1 \mathrm{~m} 7$ and Im7-tagged proteins. Unspecific binding is checked by injection of PsbV on a reference surface with immobilized Im7, whereas the sum of specific and unspecific binding is monitored on an active surface containing the Im7-tagged domain in the required amount. The reference-subtracted binding responses for interaction analysis between PSII domains and PsbV, PsbO or CyanoP are shown in Figures 3-5.
(Shen and Inoue, 1993). PsbO shows main contacts to CP43, D1a, D2, and CP47 according to the PSII crystal structure (Umena et al., 2011). The results of the SPR analysis were again in agreement with the binding position of PsbO derived from the PSII crystal structure. The PsbO subunit shows a concentration dependent and reproducible binding to immobilized $\operatorname{Im} 7-\mathrm{CP} 43\left(K_{\mathrm{D}} \approx 50 \mu \mathrm{M}\right)$, $\operatorname{Im} 7-\mathrm{D} 1 \mathrm{a}\left(K_{\mathrm{D}} \approx 50-100 \mu \mathrm{M}\right)$, and $\operatorname{Im} 7-\mathrm{D} 2\left(K_{\mathrm{D}} \approx 50 \mu \mathrm{M}\right)$ based on precisely fitted data (Figure 3) but not to Im7-CP47 and Im7PsbE. The latter result of the control experiment is reasonable, as there are no contacts between PsbE and PsbO predicted from the PSII crystal structure (Umena et al., 2011). The undetectable binding of PsbO to Im7-CP47 might be explained by the small binding area between both proteins. In summary, the successful identification of PSII subunits with significant contribution to the PsbV and PsbO association shows the potential of this SPR based approach, although minor interaction interfaces - for instance PsbV with D1 and PsbO with CP47 - might be missed in the concentration range suitable for biomolecular interaction analysis.

\section{ASSOCIATION OF CYanOP WITH PSII IS DRIVEN BY ITS INTERACTION WITH THE C-TERMINAL DOMAIN OF D2 AND THE D1 a-LOOP}

CyanoP was probed for interaction with various luminal PSII domains (CP43, CP47, mD1, D1pep, pD1, D1a, D2, and PsbE) and other putative binding partners (CyanoQ, PsbO, PsbV, PsbU, Psb27) by SPR (Figures 4 and 5; Figure S4). Besides PsbE (Figure S4), all luminal domains showed clear and reproducible binding responses, as judged by the overlay of triplicate sensograms for each concentration (Figures 4 and 5). The equilibrium responses are in excellent agreement with the fit to a one site binding isotherm yielding SEs of 

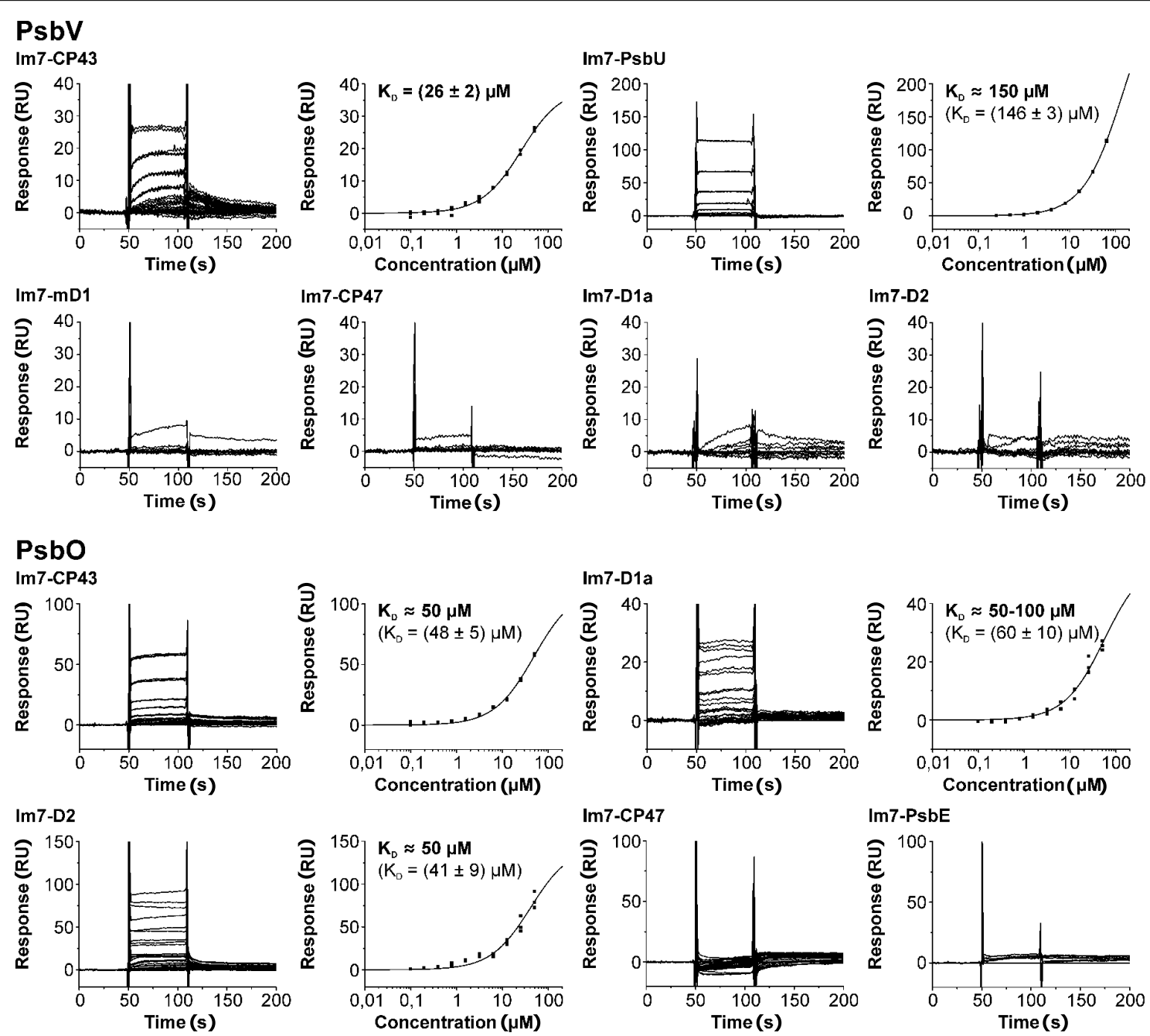

FIGURE 3 | Positive and negative controls for mapping the binding sites of soluble proteins at PSII by SPR interaction analysis.

Experiments were carried out with the known interaction partners of PsbV and PsbO derived from the PSII crystal structure (Umena etal., 2011; PsbV: CP43, mD1 and PsbU; PsbO: CP43, D1a, D2, and CP47). With two exceptions (PsbV and $\mathrm{mD} 1, \mathrm{PsbO}$ and $\mathrm{CP} 47$ ) triplicate sensograms for each analyte concentration reveal reproducible,

concentration dependent steady state binding responses (left) that can be fitted to a one site binding isotherm (right, $K_{D}$ given with SEs). Although

$5.5 \%$ of the dissociation constant. The highest affinities were measured between CyanoP and the C-terminal domain of D2 $(5.2 \pm 0.2 \mu \mathrm{M})$ and D1a $(16.2 \pm 0.9 \mu \mathrm{M})$ - both parts are located in the center of the complex (Figure 6A). A considerably lower affinity was determined for the flanking e-loops of CP43 $(55 \pm 1 \mu \mathrm{M})$ and $\mathrm{CP} 47(91 \pm 5 \mu \mathrm{M})$, for pD1 $(76 \pm 1 \mu \mathrm{M}), \mathrm{mD} 1(74 \pm 2 \mu \mathrm{M})$, and D1pep $\left(K_{\mathrm{D}} \approx 100\right.$ $150 \mu \mathrm{M})$. Within the range of error, these values indicate that the C-terminal extension of $\mathrm{pD} 1$ neither stabilizes nor prevents the association of CyanoP. Based on reconstitution experiments and cross-linking data, PsbP was proposed to interact with both $\mathrm{PsbO}$ and PsbQ in higher plants and green algae (Bricker and Frankel, 2003; Nagao et al., 2010a). Accordingly, we investigated each fit is in excellent agreement with the experimental data, most $K_{D}$ values can only be considered as an approximation, as the highest analyte concentration is smaller than the calculated $K_{D}\left(K_{D}\right.$ values determined by the evaluation software are given in parenthese for these experiments). In the experiment with PsbV and CP43 one curve was omitted due to an instrumental error (Figure S3). Additionally, negative controls with non-interacting PSII domains were performed (PsbO and PsbE, PsbV and CP47, D1a or mD1, respectively). As expected no or negligible binding was observed. interactions of CyanoP with the extrinsic cyanobacterial PSII subunits CyanoQ, PsbO, PsbV, PsbU, and Psb27, a factor involved in assembly and disassembly of PSII (Nowaczyk etal., 2006; Grasse etal., 2011). Although protein concentrations of up to $100 \mu \mathrm{M}$ were used, no interaction was observed in these experiments (Figure S4). In conclusion, the CyanoP binding site was localized at the position of PsbO on the luminal PSII surface (Figures 6B,C).

\section{CyanoP SELECTIVELY INTERACTS WITH A PSII ASSEMBLY INTERMEDIATE}

For further analysis of the interaction between PSII and CyanoP we tested binding of recombinant CyanoP to highly active dimeric 


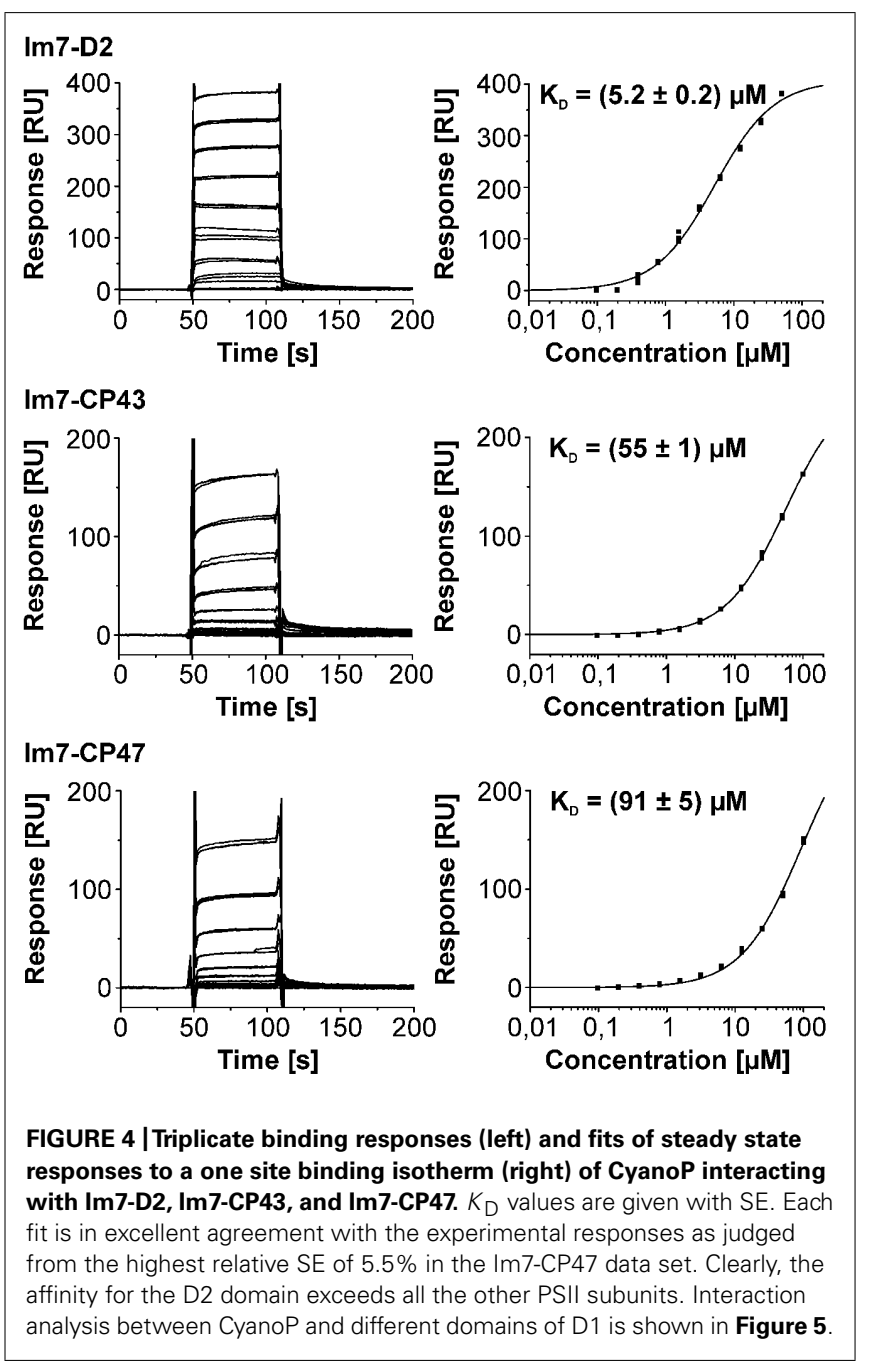

and inactive monomeric PSII from T. elongatus. In contrast to the dimer, which represents the main population of PSII and contains the extrinsic PsbO, PsbV, and PsbU subunits, the inactive monomer is associated to PSII assembly (Nowaczyk et al., 2006; Nowaczyk et al., 2012) and contains only Psb27 as extrinsic protein factor, which is associated to CP43 in close proximity to the binding site of PsbV (Liu et al., 2011). Figure 7 shows the composition of the complexes before and after reconstitution with CyanoP. The active dimer retained only trace amounts of CyanoP whereas binding to the monomeric assembly intermediate is stoichiometric. These results strongly support our previous conclusion that the extrinsic subunits and especially PsbO block the binding site of CyanoP.

\section{DISCUSSION}

Based on the results of the SPR strategy developed in this study CyanoP was precisely localized at the same position as PsbO in the PSII crystal structure (Umena et al., 2011). In addition, selective binding of CyanoP to a PSII assembly intermediate was shown by in vitro reconstitution experiments. These findings do not only shed light on the structure of CyanoP-PSII complexes but also on the function of this formerly enigmatic protein: as PsbO was shown to be specific for the main subfraction of oxygenevolving cyanobacterial PSII complexes (Nowaczyk et al., 2006), CyanoP can only be part of transient PSII species related to assembly and/or repair - a hypothesis that was discussed already (Thornton et al., 2004; Nagarajan and Burnap, 2012; Bricker et al., 2013). Although the effects of CyanoP-deletion in Synechocystis sp. PCC 6803 are limited (Thornton et al., 2004; Ishikawa et al., 2005; Summerfield et al., 2005; Sveshnikov et al., 2007) there are some indications for this proposed function: PPL1, the closest homolog of CyanoP in the PsbP superfamily of green algae and higher plants (Sato, 2010), was shown to be required for efficient PSII assembly and repair under high light stress (Ishihara et al., 2007). Unfortunately, this effect was neither reported nor excluded for any of the CyanoP deletion mutants, but notably Eaton-Rye and coworkers (Summerfield et al., 2005) noticed reduced growth under elevated temperature, a condition known to cause inactivation of PSII (Allakhverdiev et al., 2007). In concordance, a variation of charge separation properties, which was noticed in a comparative study of the different CyanoP deletion mutants (Sveshnikov et al., 2007), might also be attributed to improperly assembled PSII reaction centers (RCs) as discussed recently (Bricker et al., 2013).

Our results - in particular the interaction with CP43 - support a role of CyanoP in the late phase of PSII biogenesis as binding of CP43 is believed to be the latest step in the assembly of the membrane intrinsic part (Komenda et al., 2012). Also, the association of CyanoP with the thylakoid membrane (Ishikawa et al., 2005) argues for it having a role in late PSII biogenesis as in the early phase PSII was shown to be localized in the plasma membrane (Zak et al., 2001) or in specific thylakoid centers (Stengel et al., 2012). CyanoP may be involved in metal delivery (Nagarajan and Burnap, 2012), which is supported by the presence of zinc ions in its crystal structure (Michoux et al., 2010) and reduced growth of the deletion mutant in media devoid of calcium (Thornton et al., 2004; Summerfield et al., 2005).

Interestingly, the last 13 amino acids of the $\mathrm{mD} 1 \mathrm{C}$-terminus (D1pep) alone - including H332, E333, H337, D342, and A344 that are involved in the coordination of the $\mathrm{Mn}_{4} \mathrm{O}_{5} \mathrm{Ca}$ cluster - are sufficient for a measureable interaction with CyanoP. According to our binding model this highly conserved part of $\mathrm{D} 1$ is in close proximity to a groove build by conserved residues (Jackson et al., 2012) on the surface of CyanoP (Figure 8). Together with the result that CyanoP binds to a PSII assembly intermediate, which is free of manganese (Mamedov et al., 2007), we came to the hypothesis that the free D1 C-terminus might be coordinated by CyanoP during PSII assembly and might assist the incorporation of manganese. This idea is supported by different experimental findings: (a) the assembly and photoactivation of the $\mathrm{Mn}_{4} \mathrm{O}_{5} \mathrm{Ca}$ cluster is accompanied by structural changes due to oxidation of the first manganese atom and the concomitant binding of calcium (Chen et al., 1995; Becker et al., 2011); (b) the D1 c-loop is solvent exposed during assembly of the cluster, as D170 and E189 must be accessible for binding of free manganese and calcium (Nixon and Diner, 1992). As the carboxyl group of the C-terminal D1 alanine residue (A344) is one of the calcium ligands it is reasonable that the D1 C-terminus is part of 


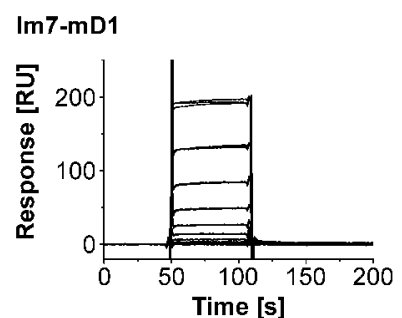

Im7-pD1

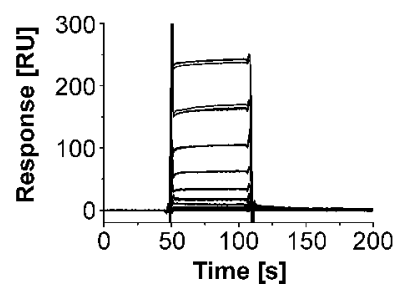

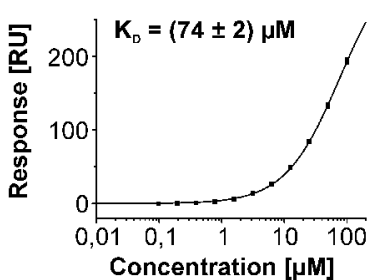

Concentration [ $\mu \mathrm{M}]$

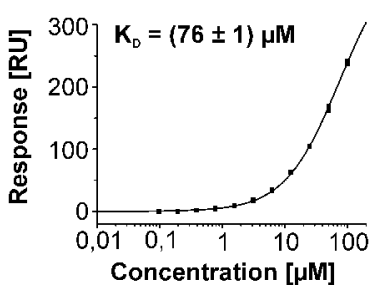

FIGURE 5 | Surface plasmon resonance interaction analysis between CyanoP and Im7 fusion proteins of pD1, mD1, D1a, and D1pep (peptide consisting of the $\mathbf{1 3} \mathbf{C}$-terminal residues of mature D1). $K_{D}$ values are given with SE. In the experiment with D1pep two curves were

\section{Im7-D1a}

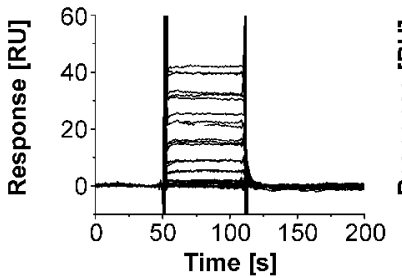

Im7-D1pep
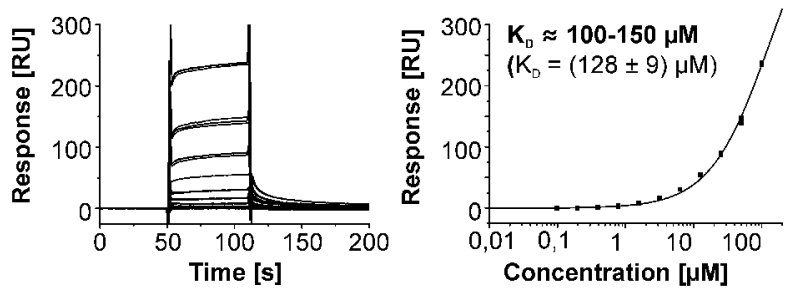

omitted due to an instrumental error (Figure S3). The affinity for D1a is the second highest measured for CyanoP. Moreover, the dissociation constants for Im7-mD1 and Im7-pD1 are - within range of error identical.

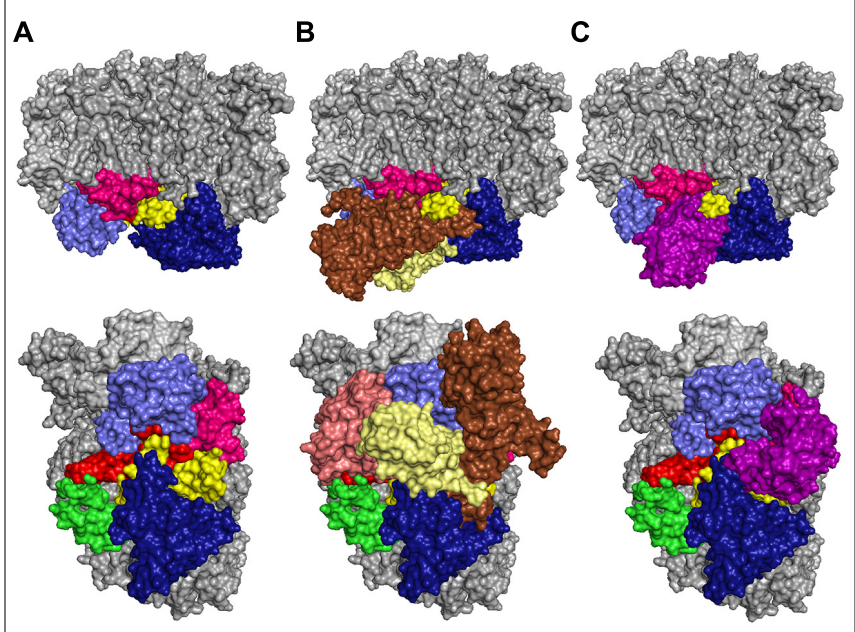

FIGURE 6 | Localization of CyanoP on PSII in side view (top) and top view (bottom). The model is based on the respective crystal structures (Michoux et al., 2010; Umena et al., 2011; pdb codes: PSII: 3ARC; CyanoP: 2XB3). (A) The PSII structure without extrinsic subunits suggests a location of CyanoP in the center to enable simultaneous contacts to the luminal domains of D1 (red), D1a (pink) D2 (yellow), CP43 (light blue) and CP47 (dark blue). The C-terminus of PsbE is shown in green. (B) The extrinsic subunits PsbO (brown), PsbV (salmon) and PsbU (pale yellow) hide most of the interaction sites in the center of the complex. (C) Positioning of CyanoP (violet) at the binding site of PsbO is the only possible model: taking into consideration that the D1 C-terminus can adopt a different conformation in premature PSII complexes lacking the oxygen-evolving complex (OEC;

Figure 8) it allows contact to all luminal domains which showed significant binding of CyanoP.

the calcium induced structural change; (c) the fast assembly of the cluster after incorporation and oxidation of the second manganese atom is based on the fact that the protein ligands are already in their final conformation (Tamura and Cheniae, 1987). The role

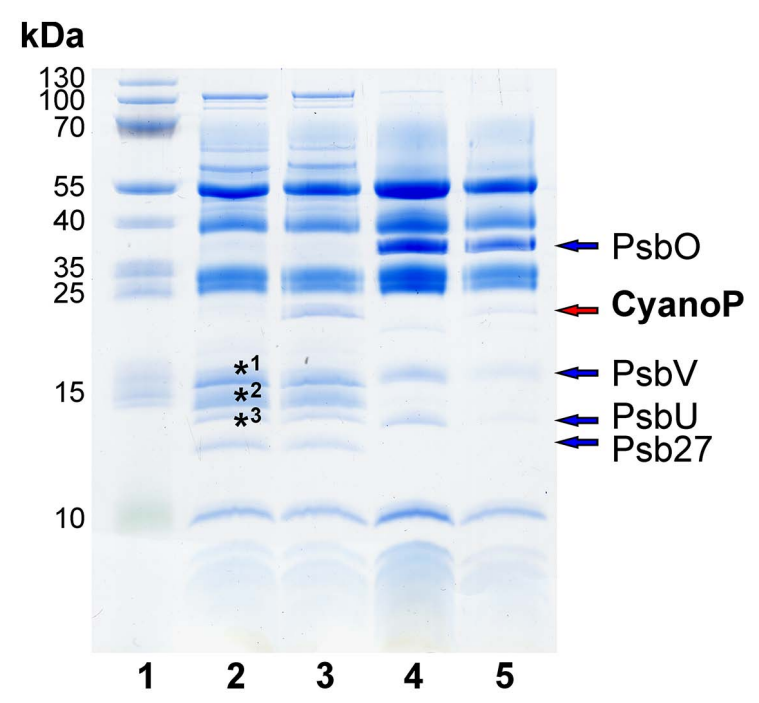

FIGURE 7 | Reconstitution of inactive monomeric and active dimeric PSII with CyanoP. CyanoP is bound in nearly stoichiometric amounts to the monomer, whereas binding to the dimeric complex is negligible. Labeled bands were identified as Psb28 (*3) and ApcA, ApcB, CpcA, CpcB, $\left({ }^{*} 1,{ }^{*} 2\right)$ by mass spectrometry (Table S2). (lane 1: molecular weight marker; lane 2 and 3: PSII monomer before and after reconstitution with CyanoP; lane 4 and 5: PSII dimer before and after reconstitution with CyanoP).

of CyanoP in this process might be to assist the incorporation of calcium by binding to the D1 C-terminus in order to keep the calcium-binding site accessible. The binding of calcium and the simultaneous structural change of the D1 C-terminus could trigger the affinity of CyanoP that would explain its release from the PSII complex during assembly.

Additionally, CyanoP might facilitate the incorporation of CP47 and CP43 into the PSII complex. Taking into account that 


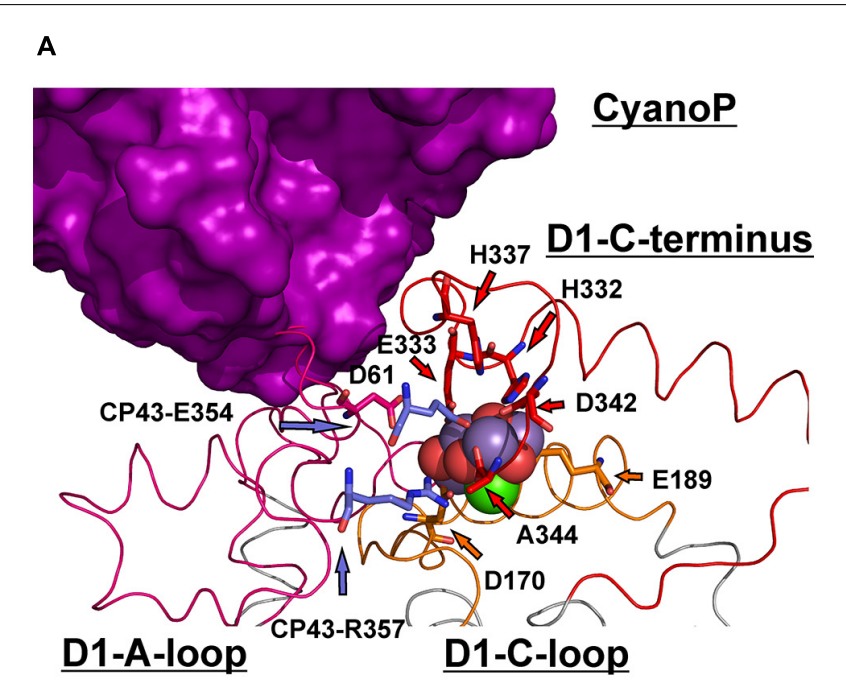

B

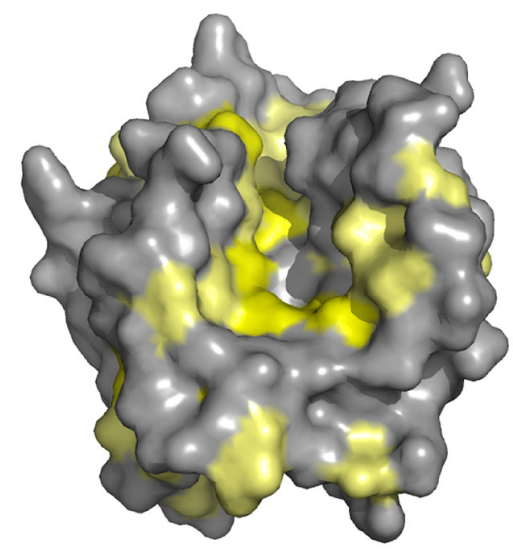

FIGURE 8 | Implications of the interaction between CyanoP and the D1 C-terminus. (A) Comparison of the suggested position of CyanoP with the coordination environment of the OEC. D1 is shown in ribbon presentation (a-loop: pink, c-loop: orange, C-terminus: red, transmembrane helices: gray). The ligands including the two residues of CP43 are depicted as sticks, whereas the atoms of the cluster are shown as spheres (oxygen: red, manganese: purple, calcium green). The $13 \mathrm{C}$-terminal residues of D1 are wound around the OEC and bury the c-loop. If this region adopts a more outstretched conformation in the absence of the OEC, a broad interaction interface with CyanoP is possible. Moreover, the c-loop can be accessed by free metal ions required for the assembly of the OEC. (B) Analysis of conserved residues on the surface of CyanoP (Michoux et al., 2010; pdb code: 2XB3) based on a previous study (Jackson et al., 2012). The Consurf-Server (Landau et al., 2005) identified a highly conserved groove on the surface of CyanoP representing an ideal binding site for the D1 C-terminus. Residues with the first and second highest degree of conservation are shown in yellow, the third highest degree in pale yellow and all other residues in gray.

the RC complex is built by D1 and D2 in the early phase of PSII biogenesis (Komenda et al., 2012), and that these subunits provide the domains with the highest affinity for CyanoP, its binding to the RC complex seems likely. The additional interactions of CyanoP with CP47 and CP43 might facilitate their sequential association to the RC complex to form RC47 (comprising D1, D2, and CP47) and subsequently the monomeric PSII-Psb27 complex (comprising D1, D2, CP47, CP43, and Psb27 as additional extrinsic assembly factor). However, this suggestion requires also further investigation

In this study we have shown that our experimental setup small-scale expression of solvent-exposed fragments or domains of membrane intrinsic subunits, direct purification on sensor surfaces and SPR interaction analysis with their soluble binding partners - allows precise identification of the protein factor binding sites. As this approach is solely based on recombinant proteins and thereby overcomes problems caused by complex instability, low abundance or transient nature, it is expected that this methodology will be used to analyze the interaction network of other transmembrane protein complexes with their soluble binding partners.

\section{ACKNOWLEDGMENTS}

We thank Dr. Olan Dolezal, Dr. Masako Iwai, and Dr. Oliver Lenz for providing the plasmids pQE30DNaseE7/Im7, PCRscript_A2_KO and PEC86, respectively. We also thank Katrin Wiegand for fruitful discussions regarding the PsbV expression, Ursula Altenfeld, Claudia König, and Melanie Völkel for excellent technical assistance and Dr. Nicholas Cox, Prof. Dr. Eckhard Hofmann, and Dr. Nicolas Plumeré for critical discussions about the manuscript. This work was supported by grants of the Ruhr-University Research School and the Greifstiftung (Kai U. Cormann), the German Federal Ministry for Education and Research, BMBF (Matthias Rögner) and by the Cluster of Excellence RESOLV (EXC 1069) funded by the Deutsche Forschungsgemeinschaft (Marc M. Nowaczyk, Matthias Rögner) and by the DFG Research Group (FOR 2092) funded by the Deutsche Forschungsgemeinschaft (Marc M. Nowaczyk).

\section{SUPPLEMENTARY MATERIAL}

The Supplementary Material for this article can be found online at: http://www.frontiersin.org/journal/10.3389/fpls.2014.00595/ abstract

\section{REFERENCES}

Allakhverdiev, S. I., Los, D. A., Mohanty, P., Nishiyama, Y., and Murata, N. (2007). Glycinebetaine alleviates the inhibitory effect of moderate heat stress on the repair of photosystem II during photoinhibition. Biochim. Biophys. Acta 1767, 13631371. doi: 10.1016/j.bbabio.2007.10.005

Anbudurai, P. R., Mor, T. S., Ohad, I., Shestakov, S. V., and Pakrasi, H. B. (1994). The ctpA gene encodes the C-terminal processing protease for the D1 protein of the photosystem II reaction center complex. Proc. Natl. Acad. Sci. U.S.A. 91, 8082-8086. doi: 10.1073/pnas.91.17.8082

Andrews, H., Li, Z., Altuve-Blanco, A., Rivera, M., and Burnap, R. L. (2005). Expression, mutagenesis, and characterization of recombinant lowpotential cytochrome c550 of photosystem II. Biochemistry 44, 6092-6100. doi: 10.1021/bi0500473

Becker, K., Cormann, K. U., and Nowaczyk, M. M. (2011). Assembly of the wateroxidizing complex in photosystem II. J. Photochem. Photobiol. B 104, 204-211. doi: 10.1016/j.jphotobiol.2011.02.005

Bricker, T. M., and Frankel, L. K. (2003). Carboxylate groups on the manganesestabilizing protein are required for efficient binding of the $24 \mathrm{kDa}$ extrinsic protein to photosystem II. Biochemistry 42, 2056-2061. doi: 10.1021/bi0 20652v

Bricker, T. M., Roose, J. L., Zhang, P., and Frankel, L. K. (2013). The PsbP family of proteins. Photosynth. Res. 116, 235-250. doi: 10.1007/s11120-0139820-7 
Chen, C., Kazimir, J., and Cheniae, G. M. (1995). Calcium modulates the photoassembly of photosystem II (Mn)4-clusters by preventing ligation of nonfunctional high-valency states of manganese. Biochemistry 34, 13511-13526. doi: 10.1021/bi00041a031

Enami, I., Murayama, H., Ohta, H., Kamo, M., Nakazato, K., and Shen, J. R. (1995). Isolation and characterization of a Photosystem II complex from the red alga Cyanidium caldarium: association of cytochrome c-550 and a $12 \mathrm{kDa}$ protein with the complex. Biochim. Biophys. Acta 1232, 208-216. doi: 10.1016/00052728(95)00122-0

Grasse, N., Mamedov, F., Becker, K., Styring, S., Rogner, M., and Nowaczyk, M. M. (2011). Role of novel dimeric Photosystem II (PSII)-Psb27 protein complex in PSII repair. J. Biol. Chem. 286, 29548-29555. doi: 10.1074/jbc.M111.238394

Hosse, R. J., Tay, L., Hattarki, M. K., Pontes-Braz, L., Pearce, L. A., Nuttall, S. D., et al. (2009). Kinetic screening of antibody-Im7 conjugates by capture on a colicin E7 DNase domain using optical biosensors. Anal. Biochem. 385, 346-357. doi 10.1016/j.ab.2008.11.026

Ido, K., Kakiuchi, S., Uno, C., Nishimura, T., Fukao, Y., Noguchi, T., et al. (2012). The conserved His-144 in the PsbP protein is important for the interaction between the PsbP N-terminus and the Cyt b559 subunit of photosystem II. J. Biol. Chem. 287, 26377-26387. doi: 10.1074/jbc.M112.385286

Ifuku, K., Nakatsu, T., Kato, H., and Sato, F. (2004). Crystal structure of the PsbP protein of photosystem II from Nicotiana tabacum. EMBO Rep. 5, 362-367. doi: 10.1038/sj.embor.7400113

Ifuku, K., Yamamoto, Y., Ono, T. A., Ishihara, S., and Sato, F. (2005). PsbP protein, but not PsbQ protein, is essential for the regulation and stabilization of photosystem II in higher plants. Plant Physiol. 139, 1175-1184. doi: 10.1104/pp.105.068643

Ishihara, S., Takabayashi, A., Ido, K., Endo, T., Ifuku, K., and Sato, F. (2007). Distinct functions for the two PsbP-like proteins PPL1 and PPL2 in the chloroplast thylakoid lumen of Arabidopsis. Plant Physiol. 145, 668-679. doi: 10.1104/pp.107.105866

Ishikawa, Y., Schroder, W. P., and Funk, C. (2005). Functional analysis of the PsbPlike protein (sll1418) in Synechocystis sp. PCC 6803. Photosynth. Res. 84, 257-262. doi: 10.1007/s11120-005-04778

Jackson, S. A., Hinds, M. G., and Eaton-Rye, J. J. (2012). Solution structure of CyanoP from Synechocystis sp. PCC 6803: new insights on the structural basis for functional specialization amongst PsbP family proteins. Biochim. Biophys. Acta 1817, 1331-1338. doi: 10.1016/j.bbabio.2012.02.032

Kakiuchi, S., Uno, C., Ido, K., Nishimura, T., Noguchi, T., Ifuku, K., et al. (2012) The PsbQ protein stabilizes the functional binding of the PsbP protein to photosystem II in higher plants. Biochim. Biophys. Acta 1817, 1346-1351. doi: 10.1016/j.bbabio.2012.01.009

Ko, T. P., Liao, C. C., Ku, W. Y., Chak, K. F., and Yuan, H. S. (1999). The crystal structure of the DNase domain of colicin E7 in complex with its inhibitor Im7 protein. Structure 7, 91-102. doi: 10.1016/S0969-2126(99)80012-4

Komenda, J., Sobotka, R., and Nixon, P. J. (2012). Assembling and maintaining the Photosystem II complex in chloroplasts and cyanobacteria. Curr. Opin. Plant Biol. 15, 245-251. doi: 10.1016/j.pbi.2012.01.017

Kuhl, H., Kruip, J., Seidler, A., Krieger-Liszkay, A., Bunker, M., Bald, D., et al (2000). Towards structural determination of the water-splitting enzyme. Purification, crystallization, and preliminary crystallographic studies of photosystem II from a thermophilic cyanobacterium. J. Biol. Chem. 275, 20652-20659. doi: 10.1074/jbc.M001321200

Landau, M., Mayrose, I., Rosenberg, Y., Glaser, F., Martz, E., Pupko, T., et al. (2005). ConSurf 2005: the projection of evolutionary conservation scores of residues on protein structures. Nucleic Acids Res. 33, W299-W302. doi: 10.1093/nar/gki370

Liu, H., Huang, R. Y., Chen, J., Gross, M. L., and Pakrasi, H. B. (2011). Psb27, a transiently associated protein, binds to the chlorophyll binding protein CP43 in photosystem II assembly intermediates. Proc. Natl. Acad. Sci. U.S.A. 108, 18536-18541. doi: 10.1073/pnas.1111597108

Mamedov, F., Nowaczyk, M. M., Thapper, A., Rogner, M., and Styring, S. (2007). Functional characterization of monomeric photosystem II core preparations from Thermosynechococcus elongatus with or without the Psb27 protein. Biochemistry 46, 5542-5551. doi: 10.1021/bi7000399

Michoux, F., Takasaka, K., Boehm, M., Nixon, P. J., and Murray, J. W. (2010). Structure of CyanoP at $2.8 \mathrm{~A}$ : implications for the evolution and function of the PsbP subunit of photosystem II. Biochemistry 49, 7411-7413. doi: 10.1021/bi1011145

Murata, N., Miyao, M., Omata, T., Matsunami, H., and Kuwabara, T. (1984). Stoichiometry of components in the photosynthetic oxygen evolution system of
photosystem-Ii particles prepared with triton X-100 from spinach-chloroplasts. Biochim. Biophys. Acta 765, 363-369. doi: 10.1016/0005-2728(84)90177-4

Nagao, R., Suzuki, T., Okumura, A., Niikura, A., Iwai, M., Dohmae, N., et al. (2010a). Topological analysis of the extrinsic PsbO, PsbP and PsbQ proteins in a green algal PSII complex by cross-linking with a water-soluble carbodiimide. Plant Cell Physiol. 51, 718-727. doi: 10.1093/pcp/pcq042

Nagao, R., Tomo, T., Noguchi, E., Nakajima, S., Suzuki, T., Okumura, A., et al. (2010b). Purification and characterization of a stable oxygen-evolving Photosystem II complex from a marine centric diatom, Chaetoceros gracilis. Biochim. Biophys. Acta 1797, 160-166. doi: 10.1016/j.bbabio.2009.09.008

Nagarajan, A., and Burnap, R. L. (2012). "Patterns of conservation and divergence of the photosystem II complex," in Functional Genomics and Evolution of Photosynthetic Systems, eds R. L. Burnap and W. F. J. Vermaas (New York: Springer), 317-344. doi: 10.1093/aob/mcs282

Nixon, P. J., and Diner, B. A. (1992). Aspartate 170 of the photosystem II reaction center polypeptide D1 is involved in the assembly of the oxygenevolving manganese cluster. Biochemistry 31, 942-948. doi: 10.1021/bi001 $18 \mathrm{a} 041$

Nowaczyk, M. M., Hebeler, R., Schlodder, E., Meyer, H. E., Warscheid, B., and Rogner, M. (2006). Psb27, a cyanobacterial lipoprotein, is involved in the repair cycle of photosystem II. Plant Cell 18, 3121-3131. doi: 10.1105/tpc.106. 042671

Nowaczyk, M. M., Krause, K., Mieseler, M., Sczibilanski, A., Ikeuchi, M., and Rogner, M. (2012). Deletion of psbJ leads to accumulation of Psb27-Psb28 photosystem II complexes in Thermosynechococcus elongatus. Biochim. Biophys. Acta 1817, 1339-1345. doi: 10.1016/j.bbabio.2012.02.017

Nowaczyk, M. M., Wulfhorst, H., Ryan, C. M., Souda, P., Zhang, H., Cramer, W. A., et al. (2011). NdhP and NdhQ: two novel small subunits of the cyanobacterial NDH-1 complex. Biochemistry 50, 1121-1124. doi: 10.1021/bil 02044b

Rengstl, B., Oster, U., Stengel, A., and Nickelsen, J. (2011). An intermediate membrane subfraction in cyanobacteria is involved in an assembly network for Photosystem II biogenesis. J. Biol. Chem. 286, 21944-21951. doi: 10.1074/jbc.M111.237867

Roose, J. L., Kashino, Y., and Pakrasi, H. B. (2007). The PsbQ protein defines cyanobacterial Photosystem II complexes with highest activity and stability. Proc. Natl. Acad. Sci. U.S.A. 104, 2548-2553. doi: 10.1073/pnas.0609 337104

Sato, N. (2010). Phylogenomic and structural modeling analyses of the PsbP superfamily reveal multiple small segment additions in the evolution of photosystem II-associated PsbP protein in green plants. Mol. Phylogenet. Evol. 56, 176-186. doi: 10.1016/j.ympev.2009.11.021

Shen, J. R., Ikeuchi, M., and Inoue, Y. (1992). Stoichiometric association of extrinsic cytochrome c550 and $12 \mathrm{kDa}$ protein with a highly purified oxygen-evolving photosystem II core complex from Synechococcus vulcanus. FEBS Lett. 301, 145149. doi: 10.1016/0014-5793(92)81235-E

Shen, J. R., and Inoue, Y. (1993). Binding and functional properties of two new extrinsic components, cytochrome c-550 and a $12-\mathrm{kDa}$ protein, in cyanobacterial photosystem II. Biochemistry 32, 1825-1832. doi: 10.1021/bi000 $58 \mathrm{a} 017$

Stengel, A., Gugel, I. L., Hilger, D., Rengstl, B., Jung, H., and Nickelsen, J. (2012). Initial steps of photosystem II de novo assembly and preloading with manganese take place in biogenesis centers in Synechocystis. Plant Cell 24, 660-675. doi: $10.1105 /$ tpc.111.093914

Summerfield, T. C., Winter, R. T., and Eaton-Rye, J. J. (2005). Investigation of a requirement for the PsbP-like protein in Synechocystis sp. PCC 6803. Photosynth. Res. 84, 263-268. doi: 10.1007/s11120-004-6431-3

Suzuki, T., Minagawa, J., Tomo, T., Sonoike, K., Ohta, H., and Enami, I. (2003). Binding and functional properties of the extrinsic proteins in oxygenevolving photosystem II particle from a green alga, Chlamydomonas reinhardtii having his-tagged CP47. Plant Cell Physiol. 44, 76-84. doi: 10.1093/pcp/ pcg010

Sveshnikov, D., Funk, C., and Schroder, W. P. (2007). The PsbP-like protein (sll1418) of Synechocystis sp. PCC 6803 stabilises the donor side of Photosystem II. Photosynth. Res. 93, 101-109. doi: 10.1007/s11120-00791713

Tamura, N., and Cheniae, G. (1987). Photoactivation of the Water-Oxidizing Complex in Photosystem-Ii Membranes Depleted of Mn and Extrinsic Proteins.1. 
Biochemical and Kinetic Characterization. Biochim. Biophys. Acta 890, 179-194. doi: 10.1016/0005-2728(87)90019-3

Thornton, L. E., Ohkawa, H., Roose, J. L., Kashino, Y., Keren, N., and Pakrasi, H. B. (2004). Homologs of plant PsbP and PsbQ proteins are necessary for regulation of photosystem ii activity in the cyanobacterium Synechocystis 6803. Plant Cell 16, 2164-2175. doi: 10.1105/tpc.104.023515

Umena, Y., Kawakami, K., Shen, J. R., and Kamiya, N. (2011). Crystal structure of oxygen-evolving photosystem II at a resolution of 1.9 A. Nature 473, 55-60. doi: 10.1038 /nature09913

Yi, X., Hargett, S. R., Liu, H., Frankel, L. K., and Bricker, T. M. (2007). The PsbP protein is required for photosystem II complex assembly/stability and photoautotrophy in Arabidopsis thaliana. J. Biol. Chem. 282, 24833-24841. doi: 10.1074/jbc.M705011200

Zak, E., Norling, B., Maitra, R., Huang, F., Andersson, B., and Pakrasi, H. B. (2001). The initial steps of biogenesis of cyanobacterial photosystems occur in plasma membranes. Proc. Natl. Acad. Sci. U.S. A. 98, 13443-13448. doi: $10.1073 /$ pnas. 241503898
Conflict of Interest Statement: The authors declare that the research was conducted in the absence of any commercial or financial relationships that could be construed as a potential conflict of interest.

Received: 31 July 2014; accepted: 13 October 2014; published online: 05 November 2014.

Citation: Cormann KU, Bartsch M, Rögner M and Nowaczyk MM (2014) Localization of the CyanoP binding site on photosystem II by surface plasmon resonance spectroscopy. Front. Plant Sci. 5:595. doi: 10.3389/fpls.2014.00595

This article was submitted to Plant Physiology, a section of the journal Frontiers in Plant Science.

Copyright (C) 2014 Cormann, Bartsch, Rögner and Nowaczyk. This is an open-access article distributed under the terms of the Creative Commons Attribution License (CC BY). The use, distribution or reproduction in other forums is permitted, provided the original author(s) or licensor are credited and that the original publication in this journal is cited, in accordance with accepted academic practice. No use, distribution or reproduction is permitted which does not comply with these terms. 